\title{
Discurso narrativo en preescolares con Trastorno Específico del Lenguaje y con desarrollo típico
}

\author{
Narrative Discourse in preschool children with \\ Specific Language Impairment and typically \\ developing children
}

Syndia Núñez G.

Mg. en Educación Especial y

Psicopedagogía

Universidad Autónoma de Chile

Maribel Granada A.

Doctora en Filosofía y Ciencias de la Educación

Universidad Católica del Maule

Francisca Cáceres Z.

Doctora en Ciencias Humanas,

Mención Discurso y Cultura

Universidad Católica del Maule

María Pilar Pomés C. PhD en Filosofía, Educación Especial, Intervención Temprana Universidad Católica del Maule

\section{RESUMEN}

En esta investigación se realizó un análisis de la producción y comprensión del discurso en preescolares de la comuna de Talca, Séptima región de Chile. El objetivo fue comparar el desempeño en la producción y comprensión del discurso narrativo de pre escolares con Trastorno Específico de Lenguaje con el desempeño de preescolares que presentan desarrollo típico. Para ello, se evaluó a niñas y niños que se encontraban cursando primer nivel de transición en una escuela de lenguaje y párvulos de la comuna de Talca. Este estudio presenta un enfoque cuantitativo con un diseño descriptivo - explicativo. La muestra contó con 40 estudiantes, 20 con Trastorno específico del lenguaje y 20 con desarrollo típico. Al comparar ambos grupos, los resultados indican que no se aprecian diferencias significativas en la producción narrativa entre ambos grupos de niños, sin embargo, sí se presentan diferencias significativas en la comprensión del discurso, a favor de los niños con desarrollo típico de lenguaje.

Palabras clave: discurso narrativo, comprensión narrativa, producción narrativa, Trastorno específico del lenguaje

\begin{abstract}
This study considered the analysis of the production and comprehension of narrative discourse by a group of preschool children in Talca, Chile. The main objective was to compare the production and comprehension of narrative discourse by preschool children with SLI and typically developing children. All children were assessed on both skills. The study adopted a descriptivecomparative design. The sample comprised 40 children: 20 children with SLI and 20 with TLD. After comparing both groups, results showed no significant differences on narrative discourse production. However, significant differences were found in narrative comprehension: children with TLD performed higher than children with SLI.
\end{abstract}

Keywords: narrative discourse, narrative discourse comprehension, narrative discourse production, Specific Language Impairment
Autor correspondiente Syndia Núñez G. Santiago - Chile Correo-e: snunezg@uautonoma.cl

Recibido: 03/07/2017 Aceptado: $18 / 10 / 2017$ 


\section{Introducción}

Las primeras experiencias escolares son relevantes para despertar el interés por aprender a relacionarse verbalmente. La acogida, la participación, la colaboración y el respeto a la diversidad son condiciones necesarias para que las interacciones orales en el aula sean de calidad. En este sentido el discurso narrativo cobra importancia en tanto organizador de las experiencias de niños y niñas, permitiendo tomar conciencia de los hechos de una situación temporal y espacial real (Brunner, 1990). Así en una primera instancia el niño usa el lenguaje para satisfacer necesidades, luego comienza a elaborar sus producciones para comunicarse, ya no solo con sus seres más cercanos, sino que también con sus pares y la comunidad educativa a la cual se integra al momento de entrar a la etapa preescolar.

Por otra parte, según Rosemberg, Menti, Stein, Alam y Migdalek (2016) las narrativas sobre eventos pasados y futuros permiten a los niños entender que los eventos tienen una secuencia temporal. Para generar estas narraciones, los niños deben recordar una experiencia y representarla verbalmente estableciendo relaciones con la lengua.

Se entiende por discurso narrativo a un conjunto de oraciones organizadas coherentemente que alude a una sucesión de acontecimientos, relacionados temporal y causalmente, y caracterizados por su complicación y orientación hacia una resolución (Bassols y Torrent, 1997).

Sin duda la capacidad narrativa es un proceso que se desarrolla a medida que el niño amplía su crecimiento cognitivo y sus habilidades lingüísticas, ya que se requiere que pueda mantener en la mente la representación de una realidad compleja y formularla mediante el lenguaje. Debe poder expresarla con palabras adecuadas evocando un mundo distante en el tiempo y en el espacio, de manera que sea coherente y cohesionada para ser comprendida por el oyente (Cardona y Civit, 2008; López, Duque, Camargo y Ovalle, 2014).

El desarrollo del discurso narrativo se plantea como un predictor del éxito escolar, siendo una importante fuente de información del comportamiento social y lingüístico del niño (Acosta, Moreno y Axpe, 2013). Específicamente se ha relacionado con las habilidades de comprensión lectora, pues la posibilidad de comprender y producir narraciones por parte de los niños permite el desarrollo de la alfabetización y de habilidades vinculadas al rendimiento académico (Wellman, Lewis, Freebairn, Avrich, Hansen \& Stein, 2011). Por otra parte, la narrativa de los niños permite conocer su desarrollo cognitivo y lingüístico, ayudando a identificar a tiempo si se presenta un retraso o descenso en el desarrollo del lenguaje.

Algunos de los procesos cognitivos que los niños necesitan desarrollar son: memoria, atención, organización y planificación, los que serán un soporte para considerar la variedad de perspectivas en una trama y relacionar eventos causal y temporalmente. Sumado a esto, el conocimiento del mundo es un requisito previo para la conceptualización y la narración de una historia, debido a que su desarrollo implica una serie de inferencias relacionadas con el mundo cotidiano (Duinmeijer, De Jong \& Scheper, 2012).

El desarrollo del discurso narrativo en la primera infancia se ha asociado al juego y a la utilización de 
guiones, como primeras aproximaciones que permiten expresar la estructura de una historia, así el juego intencional puede ser un precursor del juego narrativo, dando paso a relatos exclusivamente verbales, a partir de sucesos cotidianos repetidos en el tiempo. De acuerdo a Applebee (1978), entre los 2 y 3 años se observa el agrupamiento enumerativo, estadio en el cual no hay un tema central ni organización, sin embargo, el niño nombra y describe enumerando sucesos. Luego a los 3 años se presenta una secuencia de acciones en torno a un personaje, lo que incluye nombrar y describir sucesos en torno a éste, un tema central y un ambiente sin eventos relacionados entre sí. Posteriormente las primeras narraciones se presentan desde los 4 a los 4 años 6 meses, periodo en que la historia tiene un núcleo central, se aprecia un hecho inicial y una consecuencia, sin embargo, no existe final. A este periodo le siguen, entre los 4, 6 meses y los 5 años, las narraciones con episodios incompletos o cadenas narrativas, para llegar luego a las narraciones verdaderas desde los 5 a los 7 años.

En este sentido es necesario el conocimiento del mundo como un requisito previo para la conceptualización y la narración de una historia, ya que las historias contienen muchas inferencias implícitas hechas sobre la base de la vida cotidiana. En el proceso de producción de narraciones, los niños pueden representar distintos roles tales como narrador, audiencia o jugador en paralelo, asumiendo un intercambio de roles entre pares y un proceso de negociación que les permite construir sus producciones (Alam y Rosemberg, 2015).

Las trayectorias de desarrollo se manifiestan en forma atípica en algunos niños, evidenciando una dificultad específicamente lingüística como es el caso de los niños que presentan Trastorno específico del lenguaje (TEL). Esta es una limitación significativa asociada a la expresión, comprensión del lenguaje o ambos (Coloma, Pavez, Peñaloza, Araya, Maggiolo y Palma, 2012), que se presenta en uno o algunos de los componentes lingüísticos. Es necesario descartar problemas sensoriales, discapacidad intelectual, graves alteraciones de la relación o la comunicación, dificultades emocionales o dificultades culturales (MINEDUC, 2009; Llorenç, Aguado, Cardona y SanzTorrent, 2014).

También los niños con TEL manifiestan problemas en diferentes ámbitos del discurso narrativo, tales como la estructura textual, la coherencia y los mecanismos de cohesión (Coloma, Sotomayor, De Barbieri y Silva, 2015). La capacidad narrativa oral es el indicador y predictor más poderoso de la presencia del TEL, ya que estos niños suelen mostrar déficits importantes en esta área y de ahí su relevancia, tanto para la detección precoz como para la intervención temprana de este trastorno (Acosta et al., 2013).

Según Maggiolo, Coloma y Pavez (2009), estudios realizados señalan que los niños con TEL desarrollan más tardíamente sus narraciones que los niños con desarrollo típico del lenguaje. Estos niños presentan dificultades para ordenar una historia, reconocer al protagonista y respetar la secuencia de los eventos. También manifiestan problemas en la coherencia y en la superestructura de sus narraciones. El hecho de que un niño en esta edad no emplee una estructura narrativa en su recontado evidencia un problema en su desarrollo psicolingüístico o una alteración del lenguaje. 
Respecto a esto, algunas investigaciones han encontrado que la competencia de comprender $y$ producir narrativas orales por parte de los niños está fuertemente relacionada con el desarrollo de habilidades de alfabetización y el rendimiento académico (Fiorentino \& Howe, 2004; Wellman et al., 2011). Asimismo, se ha sugerido que los estudiantes que presentan patrones desordenados del lenguaje y de la comunicación manifestarán una alteración y restricción tanto en su interacción social como en el aprendizaje escolar (Acosta et al., 2013). Valorar tempranamente el desarrollo del discurso narrativo permitirá disminuir la brecha existente entre niños con y sin dificultades de lenguaje al empezar su escolaridad. Lo anterior permitirá realizar una mirada preventiva tendiente a disminuir la desigualdad lingüística y evitando su agudización en el transcurso de la educación formal.

Este estudio de investigación busca comparar el desempeño de la producción y comprensión del discurso narrativo en pre escolares con TEL con el desempeño de niños con desarrollo típico de lenguaje, de la comuna de Talca. Es importante señalar que en esta localidad existen alrededor de 31 Escuelas de Lenguaje y no se cuenta con suficiente información acerca del desempeño de los niños con TEL. Es por esta razón que resulta relevante entregar información específica en el área del discurso narrativo en una muestra de estudiantes de la comuna de Talca. Con esta información, por una parte, sería factible en estudios posteriores analizar los resultados en comparación con la Región Metropolitana. Por otra parte, podría apoyarse la toma de decisiones relacionadas con prevención e intervención en pre escolares que se encuentran muy próximos a iniciar el proceso de lectura en la educación básica.

\section{Método}

La investigación tiene un enfoque cuantitativo, con un alcance descriptivo y explicativo, siendo su diseño no experimental, transeccional comparativo.

\section{Participantes}

El grupo en estudio está compuesto por 40 preescolares, 20 con desarrollo típico del lenguaje y 20 estudiantes diagnosticados con TEL, los cuales asisten a una escuela de lenguaje y de párvulos, pertenecientes al nivel de kínder, con dependencia particular subvencionada de la comuna de Talca. Las edades de los niños fluctúan entre los 5 años y 6 años 3 meses. Los criterios de selección de los participantes con desarrollo típico de lenguaje fueron los siguientes: (a) niños y niñas matriculados en kínder para párvulos sin TEL, (b) niños y niñas sin problemas sensoriales, ni cognitivos, (c) niños y niñas autorizados por los padres (tutor), para participar en el estudio, (d) niños y niñas con 5 años cumplidos al 31 de marzo del año lectivo. Los criterios de selección de los participantes con TEL fueron: (a) niños y niñas matriculados en kínder en escuelas de lenguaje; (b) niñas y niños con TEL expresivo o mixto. Cabe señalar que estos niños cuentan con un diagnóstico fonoaudiológico, con las pruebas evaluativas estandarizadas para el contexto nacional (e.g., Test para evaluar procesos de simplificación fonológica, TEPROSIF-R; Test exploratorio de gramática española, TEGE y Test de comprensión auditiva de lenguaje, TECAL); (c) niños y niñas que 
fueran autorizados por los padres (tutor) para participar en el estudio; d) niños y niñas con los 5 años cumplidos al 31 de marzo del año lectivo.

Tabla 1 Características de la muestra estudiada

\begin{tabular}{|c|c|c|}
\hline Lenguaje y género & $\mathrm{n}$ & Edad promedio \\
\hline TEL masculino & 8 & 70 meses \\
\hline TEL femenino & 12 & 70 meses \\
\hline $\begin{array}{l}\text { Desarrollo típico } \\
\text { masculino }\end{array}$ & 9 & 67 meses \\
\hline $\begin{array}{l}\text { Desarrollo típico } \\
\text { femenico }\end{array}$ & 11 & 67 meses \\
\hline
\end{tabular}

\section{Instrumentos}

Se realizó la evaluación del discurso narrativo aplicando el instrumento de Evaluación del Discurso Narrativo (EDNA) de Pavez, Coloma y Maggiolo (2008) cuyo objetivo es medir el desempeño narrativo tanto a nivel expresivo como comprensivo que Posee un índice de confiabilidad de 0.77 en la producción del discurso y de 0.84 en la comprensión del discurso, lo que evidencia niveles de confiabilidad aceptable para este tipo de instrumento (Pavez et al., 2008). Cuenta con normas estandarizadas en Chile. Su aplicación es individual en edades comprendidas entre los 3 y los 10 años. En cuanto a la producción del discurso, evalúa la superestructura: presentación, episodio y final, a través del recontado de tres cuentos. En cuanto a la comprensión narrativa este instrumento evalúa las respuestas de los niños frente a preguntas literales que refieren a tres categorías de la superestructura narrativa, presentación, episodio y final. También se valoran las interrogantes de tipo inferencial, que exigen elaborar respuestas que relacionan la información del texto con los conocimientos previos que posee el niño.
Los resultados estadísticos para comprobar la validez y confiabilidad de estas pruebas, indica que se trata de un instrumento sensible que permite discriminar la comprensión del discurso de los niños evaluados. Asimismo, se estableció una correlación significativa comprobando que a mayor edad aumenta la puntuación obtenida por los niños evaluados $(r=0.66, p=0.00)$. En relación a la confiabilidad, los estudios psicométricos de este instrumento muestran en general correlaciones significativas entre cada ítem y la puntuación total, se evidencia con esto un adecuado nivel de consistencia interna.

\section{Procedimientos}

La aplicación del EDNA se realizó de manera individual, durante una sesión de aproximadamente 30 minutos. La indicación para el estudiante era que se le leerían unos cuentos y que él después debería contarlos. Se utilizaron tres cuentos ("La ardillita glotona", "El sapito saltarín" y "El lobo friolento"). Cada narración se leía de manera natural y sin apoyo visual. El orden de la lectura fue 10 "La ardillita glotona", 2ㅇ "El sapito saltarín" y 3ㅇ "El lobo friolento". Luego de escuchar cada cuento, el niño realizaba el recontado y posteriormente respondía las preguntas de comprensión, y así sucesivamente hasta completar los tres cuentos. La sesión era grabada en audio y al finalizar se le entregaba un refuerzo positivo al niño/niña.

La corrección se realizó siguiendo los criterios establecidos por el EDNA. La producción de narraciones se efectuó asignando puntaje en base al análisis de criterios estructurales. Primero se determinó si los cuentos poseían o no estructura. 
Cuando los relatos infantiles carecieron de estructura se consignaron los siguientes puntajes: no dice nada (0 punto), dice una o dos oraciones (0.25 puntos), aglutina acciones sin relación (0.5 puntos), agrupa oraciones en torno a un personaje (0.75 puntos) o produce secuencia de acción más obstáculo u obstáculo más resultado (0.75 puntos). Para los relatos infantiles con estructura se identificó cada categoría y elementos del cuento. A cada uno de ellos se les asignó un puntaje, es así como presentación completa obtuvo 1 punto, presentación incompleta 0.5 , episodio completo 2 puntos, incompleto 1 punto, el final abrupto, 1 punto, y el normal, 2 puntos.

Por su parte, en la comprensión de narraciones se asignó 1 punto a las respuestas literales, 2 puntos a las que requieren inferencias y 0 a las incorrectas. EI hecho de que a las respuestas inferenciales tengan una mayor puntuación se debe a que el sujeto efectúa relaciones entre la información explícita del texto y su conocimiento previo.

Se estableció que los preescolares con dificultades en la producción o en la comprensión de discurso narrativo eran aquellos que se ubicaban en el percentil equivalente o inferior a 10. En producción este rendimiento corresponde a 9 puntos o menos, y en comprensión, a 19 puntos o a puntuaciones inferiores.

El análisis estadístico fue realizado a través del programa SPSS versión 23 (2016). La técnica seleccionada fue de estadística descriptiva, además de considerar la prueba t de Student, para muestras independientes.

\section{Resultados}

En este apartado se exponen los resultados en relación a la comparación del desempeño de los niños con TEL y con desarrollo típico, en función de la comprensión y expresión del discurso narrativo.

En la Tabla 2, se presentan los resultados obtenidos por los niños en la evaluación de la producción narrativa. Se puede advertir que 13 niños con desarrollo típico no estructuran su producción mientras que 7 sí lo realizan. En el caso de los niños con TEL, 17 de ellos no estructuran su producción narrativa y solo 3 sí lo hacen. Los niños con TEL presentan dificultades para lograr la estructura del relato, pues tienden a expresar una oración o aglutinan los contenidos. En cambio, los niños con desarrollo típico que no logran utilizar los componentes de la superestructura, tienden a agrupar el relato en un personaje. Fue posible observar que los niños con TEL utilizan menos recursos verbales para recontar la historia que los niños con desarrollo típico.

Tabla 2

Desempeño en la producción narrativa

\begin{tabular}{lccc}
\hline Producción & $\mathrm{n}$ & $\begin{array}{c}\text { No } \\
\text { estructuran }\end{array}$ & Estructuran \\
\hline $\begin{array}{l}\text { Desarrollo } \\
\text { típico }\end{array}$ & 20 & 13 & 7 \\
TEL & 20 & 17 & 3 \\
\hline
\end{tabular}

En la Tabla 3, se observa que 14 estudiantes con desarrollo típico obtienen un rendimiento normal, 2 disminuido y 4 deficiente. En tanto 3 niños con TEL obtienen un rendimiento normal, 4 disminuido y 13 deficiente. Se analizaron complementariamente las respuestas por preguntas literales e inferencias. 
Según lo obtenido, se logró evidenciar cuales fueron las dificultades que presentan los preescolares con TEL, donde su rendimiento fue inferior en el cuento La ardillita glotona y El sapito saltarín, en cambio, no se evidenciaron diferencias significativas en el cuento El lobo friolento donde ambos grupos lograron responder acertadamente tanto las preguntas literales como inferenciales.

Tabla 3

Desempeño en la comprensión narrativa

\begin{tabular}{lccc}
\hline Comprensión & Normal & Disminuido & Deficiente \\
\hline $\begin{array}{l}\text { Desarrollo } \\
\text { típico }\end{array}$ & 14 & 2 & 4 \\
TEL & 3 & 4 & 13 \\
\hline
\end{tabular}

En la tabla 4, se observa el desempeño de la producción de narraciones en ambos grupos. En los niños con desarrollo típico se observa una media de 6,06 y en los niños con TEL una media de 4,75.

Tabla 4

Medias de la producción narrativa

\begin{tabular}{lccc}
\hline Lenguaje & $\mathrm{n}$ & Media & $\begin{array}{c}\text { Desviación } \\
\text { estándar }\end{array}$ \\
\hline $\begin{array}{l}\text { Desarrollo } \\
\text { típico }\end{array}$ & 20 & 6.06 & 4.33 \\
TEL & 20 & 4.75 & 4.82 \\
\hline
\end{tabular}

En la tabla 5, se observa que las varianzas son iguales entre ambos grupos. La significancia es mayor a 0.05 lo que indica que no existen diferencias estadísticamente significativas entre ambos grupos.
Tabla 5

Comparación de resultados en producción narrativa de niños con y $\sin T E L$

\begin{tabular}{ccccc}
\hline$F$ & Sig. & $\begin{array}{c}\text { Sig. } \\
\text { (bilateral) }\end{array}$ & $\begin{array}{c}\text { Diferencia } \\
\text { de } \\
\text { medias }\end{array}$ & $\begin{array}{c}\text { Diferencia } \\
\text { de error } \\
\text { estándar }\end{array}$ \\
\hline 0.00 & 1.00 & .37 & 1.31 & 1.44 \\
\hline$p<.005$ & & & &
\end{tabular}

En la tabla 6, se determina el desempeño de la comprensión narrativa en preescolares con y sin TEL. En los niños con desarrollo típico se obtiene una media de 21,1 y en los niños con TEL de 13,1.

Tabla 6

Medias de la comprensión narrativa

\begin{tabular}{lccc}
\hline Lenguaje & $\mathrm{n}$ & Media & $\begin{array}{c}\text { Desviación } \\
\text { estándar }\end{array}$ \\
\hline $\begin{array}{l}\text { Desarrollo } \\
\text { típico }\end{array}$ & 20 & 21.1 & 7.20 \\
TEL & 20 & 13.1 & 6.46 \\
\hline
\end{tabular}

En la tabla 7, se presentan el desempeño de la comprensión narrativa en preescolares con y sin TEL. En este caso se observa diferencias en los puntajes promedio estableciéndose diferencias estadísticamente significativas entre ambos grupos.

Tabla 7 Comparación de resultados en comprensión narrativa de niños con y $\sin T E L$

\begin{tabular}{ccccc}
\hline$F$ & Sig. & $\begin{array}{c}\text { Sig. } \\
\text { (bilateral) }\end{array}$ & $\begin{array}{c}\text { Diferencia } \\
\text { de } \\
\text { medias }\end{array}$ & $\begin{array}{c}\text { Diferencia } \\
\text { de error } \\
\text { estándar }\end{array}$ \\
\hline 0.007 & .93 & .001 & 8.00 & 2.16 \\
\hline$p<.005$ & & & & \\
\end{tabular}

\section{Discusión}

De acuerdo a lo observado en el presente estudio, se puede señalar que en la producción 
narrativa, los niños con desarrollo típico presentaron un desempeño similar a los niños con TEL. Es posible mencionar que los resultados encontrados son coincidentes con lo señalado en otro estudio (Coloma, Mendoza y Carballo, 2017).

Los niños con y sin TEL muestran una expresión narrativa oral con un nivel de desarrollo semejante. En ambos grupos nos encontramos con niños que no utilizan la superestructura narrativa, lo que se refleja por ejemplo en carencias en la presentación de personajes y sus características, especificación del problema, la temporalidad de los hechos relatados y la determinación de las soluciones que conlleva al final del cuento.

Un estudio realizado en la Región Metropolitana en niños con TEL, muestra que el $42 \%$ de niños de 4 a 5 años presentan déficit narrativo, alrededor del $20 \%$ presenta un desarrollo disminuido y de $35 \%$ presenta un desarrollo normal (Pavez et al., 2008). En el presente trabajo, se observan resultados semejantes para la producción narrativa, puesto que los niños diagnosticados con TEL se agrupan mayoritariamente en las categoría de menor desarrollo para estas habilidades (no estructuran, disminuido y deficiente), representando al $85 \%$ de los niños con TEL, evaluados en la comuna de Talca.

Al comparar los puntajes obtenidos por los niños talquinos sin TEL con los promedios obtenidos por niños con desarrollo típico de la investigación de Pavez et al., (2008) se aprecian diferencias en el desempeño narrativo. Los niños talquinos ( $n=20 ; X$ $=6,06)$ muestran un desempeño inferior al obtenido por los niños de la muestra metropolitana ( $n=50 ; X$ $=10,8)$.
Respecto a la comprensión, los resultados muestran diferencias estadísticamente significativas entre niños con desarrollo típico y niños con TEL en comprensión narrativa, a favor de los estudiantes sin TEL. Un resultado semejante se observó en una investigación previa (Coloma et al., 2017).

Respecto a los niños con desarrollo típico, se observó que los niños de la comuna de Talca logran un puntaje 21,1 y los niños del trabajo de Pavez et al., (2008) alcanzan una puntuación promedio de 25,8 .

Los niños evaluados en este estudio se encuentran próximos a ingresar a primer año básico y comenzar con el proceso de aprendizaje de la lectura. La comprensión narrativa oral es una de las habilidades que sustenta a la comprensión lectora (Oakhil \& Cain, 2012). En consecuencia, los resultados obtenidos por los niños con TEL en comprensión narrativa podrían afectar su posterior comprensión lectora.

Cabe mencionar como una debilidad del presente estudio el hecho de que los resultados obtenidos son válidos solo para esta muestra y no pueden ser generalizados puesto que es un grupo muy pequeño, son sólo 40 preescolares. Entre las proyecciones de este estudio se encuentra la importancia de abordar el discurso narrativo desde los primeros niveles de la educación parvularia, considerando la evaluación del discurso y su estimulación, a través de planes de intervención en base a historias ficticias o reales basadas en situaciones cotidianas. Por otra parte, sería recomendable realizar estudios de la misma índole con muestras más amplias y que puedan ser extrapolables a la población en general. 


\section{Referencias}

Acosta, V., Moreno, A. y Axpe, A. (2013). Análisis de las dificultades de Discurso Narrativo en alumnado con Trastorno específico de Lenguaje. Revista de Logopedia, Foniatría y Fonoaudiología, 33, 165-171. http://dx.doi.org/10.1016/j.rlfa.2013.07.004

Alam, F. y Rosemberg, C. (2015). Un análisis del proceso de construcción interaccional en narrativas de ficción entre pares. Papeles de Trabajo sobre Cultura, Educación y Desarrollo Humano. 11(1), 1-16.

Applebee, A. (1978). The child's concept of story. Chicago: University of Chicago Press.

Brunner, J. (1990). Acts of Meaning. Cambridge,M.M.:Harvard University Press

Bassols, M. y Torrent, A. (1997) Modelos textuales. Teoría y práctica. Barcelona: Octaedro.

Cardona, M. y Civit, A. (2008). Valoración dinámica de la habilidad narrativa: aplicación en un caso de trastorno específico de lenguaje. Revista de Logopedia, Foniatría y Audiología, 28(4), 245-260.

Coloma, C., Mendoza, E. \& Carballo, C. (2017). Desempeño Gramatical y Narrativo en Niños con Trastorno Específico de Lenguaje. Círculo de Lingüística Aplicada de la Comunicación, 69, 67-90. Recuperado de: http://dx.doi.org/10.5209/CLAC.55314

Pavez M.M., Coloma, C. \& Maggiolo, M. (2008). El desarrollo narrativo en niños: Una propuesta práctica para la evaluación y la intervención en niños con trastorno del lenguaje. Barcelona: Ars Médica.

Coloma, C., Pavez, M., Peñaloza, C. Araya, C., Maggiolo, M. y Palma, S. (2012). Desempeño lector y narrativo en escolares con trastorno específico. Onomázein, 26, 351$375 . \quad$ Recuperado de: http://www.redalyc.org/articulo.oa?id=134525391013

Coloma, C., Sotomayor, C., De Barbieri, Z. y Silva, M. (2015). Comprensión lectora, habilidades lingüísticas y decodificación en escolares con TEL. Revista de Investigación en Logopedia, 5(1), 1-17. Recuperado de http://revistalogopedia.uclm.es/ojs/index.php/revista/arti cle/view/126/89
Duinmeijer, I., De Jong, J. \& Scheper, A. (2012). Narrative abilities, memory and attention in children with a specific language impairment. International Journal of Language \& Communication Disorders, 47(5), 542-555. DOI:10.1111/j.1460-6984.2012.00164.x

Fiorentino, L. \& Howe, N. (2004). Language Competence, Narrative Ability, and School Readiness in Low-Income Preschool Children. Canadian Journal of Behavioural Science, 36 (4), 280 -294. DOI:10.1037/h0087237

López, L., Duque, C., Camargo, G. y Ovalle, A. (2014). Comprensión y producción textual narrativa en preescolares. Psicología desde el Caribe, 31(1). Recuperado de:

http://rcientificas.uninorte.edu.co/index.php/psicologia/ar ticle/viewFile/5191/5226

Llorenç, A., Aguado, G., Cardona M. y Sanz-Torrent, M. (2014). El Trastorno Específico del Lenguaje: Diagnóstico e intervención. Barcelona, España: UOC.

Maggiolo, M., Coloma, C. y Pavez, C. (2009). Estimulación de narraciones infantiles. Revista CEFAC, 11(3), 379-388. Recuperado de: http://www.pdfio.com/k-2118554.html

Ministerio de Educación (MINEDUC). (2009). Decreto Supremo, 170/2009, Ley 20201, Unidad de Educación Especial.

Oakhil, J. \& Cain, K. (2012). The Precursors of Reading Ability in Young Readers: Evidence from a Four-Year Longitudinal Study. Scientific Studies of Reading, 16(2), 91121

Pavez M.M., Coloma, C. \& Maggiolo, M. (2008). El desarrollo narrativo en niños: Una propuesta práctica para la evaluación y la intervención en niños con trastorno del lenguaje. Barcelona: Ars Médica.

Rosemberg, C., Menti, A., Stein, A., Alam, F y Migdalek, M. (2016). Vocabulario, narración y argumentación en los primeros años de la infancia y la niñez. Una revisión de investigaciones. Revista Costarricense de Psicología, 35(2), 139-158.

Wellman, R., Lewis, B., Freebairn, L., Avrich, A., Hansen, A. \& Stein, C. (2011). Narrative Ability of Children with Speech Sound Disorders and the Prediction of Later Literacy Skills. Language, Speech, and Hearing Services in Schools, 42, 561-579. DOI:10.1044/0161-1461(2011/10-0038). 